\title{
ON THE IDEAL STRUCTURE OF THE TENSOR PRODUCT OF NEARLY SIMPLE ALGEBRAS
}

\author{
ILJA GOGIĆ
}

\begin{abstract}
We define a unital algebra $A$ over a field $\mathbb{F}$ to be nearly simple if $A$ contains a unique non-trivial ideal $I_{A}$ such that $I_{A}^{2} \neq\{0\}$. If $A$ and $B$ are two nearly simple algebras, we consider the ideal structure of their tensor product $A \otimes B$. The obvious non-trivial ideals of $A \otimes B$ are:

$$
I_{A} \otimes I_{B}, \quad I_{A} \otimes B, \quad A \otimes I_{B}, \quad \text { and } \quad I_{A} \otimes B+A \otimes I_{B} .
$$

The purpose of this paper is to characterize when are all non-trivial ideals of $A \otimes B$ of the above form.
\end{abstract}

\section{INTRODUCTION}

Let $A$ and $B$ be unital algebras over a field $\mathbb{F}$. If $A$ is a central simple algebra, it is well-known that all ideals of the tensor product $A \otimes B$ are of the form $A \otimes J$, where $J$ is an ideal of $B$ (see e.g. [4, Theorem 4.42] and the comment following its proof).

However, the ideal structure of $A \otimes B$ is generally much more complicated than the one of $A$ and $B$, even in the simplest cases when $A$ and $B$ are proper field extensions of $\mathbb{F}$. In fact, if $\mathbb{K}$ is any proper field extension of $\mathbb{F}$, then $\mathbb{K} \otimes_{\mathbb{F}} \mathbb{K}$ is never a field, since for any $x \in \mathbb{K} \backslash \mathbb{F}$ the non-zero tensor $1 \otimes x-x \otimes 1$ lies in the kernel of the multiplication $m: \mathbb{K} \otimes_{\mathbb{F}} \mathbb{K} \rightarrow \mathbb{K}, m(x \otimes y)=x y$. Moreover, the problem of characterizing when is the tensor product of two fields a field (or a domain) is highly non-trivial and for results on this subject we refer to [7] and the references within. We also refer to a survey paper 8 that considers which properties of commutative algebras $A$ and $B$ are conveyed to $A \otimes B$.

In this paper we study the ideal structure of the tensor product of two unital algebras that both contain only one non-trivial ideal. To avoid pathologies, we also add one additional requirement:

Definition 1.1. We define a unital algebra $A$ to be nearly simple if $A$ contains a unique non-trivial ideal, denoted by $I_{A}$, such that $I_{A}^{2} \neq\{0\}$.

The basic examples of nearly simple algebras are the unitizations of non-unital simple algebras (Example 3.2). Further, if $V$ is a vector space over $\mathbb{F}$ of countably infinite dimension, then the algebra $A=\operatorname{End}_{\mathbb{F}}(V)$ of all linear operators on $V$ is nearly simple, where $I_{A}$ is the ideal of finite rank operators (Example 3.3).

Date: January 1, 2020.

2010 Mathematics Subject Classification. Primary 15A69, 16D25 Secondary 16N60.

Key words and phrases. nearly simple algebra, tensor product of algebras, ideals.

This work has been fully supported by the Croatian Science Foundation under the project IP-2016-06-1046.

The author is grateful to Matej Brešar for his comments. 
If $A$ and $B$ are two nearly simple algebras, then the obvious non-trivial ideals of $A \otimes B$ are:

$$
I_{A} \otimes I_{B}, \quad I_{A} \otimes B, \quad A \otimes I_{B}, \quad \text { and } \quad I_{A} \otimes B+A \otimes I_{B}
$$

The main result of this paper is Theorem 3.9 , in which we characterize when are all non-trivial ideals of $A \otimes B$ of the above form.

\section{Preliminaries}

Throughout this paper $\mathbb{F}$ denotes a field. Unless specified otherwise, our vector spaces (algebras) will be over $\mathbb{F}$ and all tensor products will be over $\mathbb{F}$. Also, our algebras are assumed to be associative.

Given any algebra $A$, we write $Z(A)$ for the centre of $A$. For $x, y \in A$, the commutator $x y-y x$ is denoted by $[x, y]$. By an ideal of $A$ we always mean a two-sided ideal. As usual, we say that an ideal $I$ of $A$ is non-trivial if $I \neq\{0\}$ and $I \neq A$. If $A$ is unital and $Z(A)=\mathbb{F} 1, A$ is said to be central.

For an element $a \in A$ by $\langle a\rangle$ we denote the principle ideal generated by $a$. Further, for $a, b \in A$ we define a two-sided multiplication

$$
M_{a, b}: A \rightarrow A \quad \text { by } \quad M_{a, b}: x \mapsto a x b .
$$

By an elementary operator on $A$ we mean a map $\phi: A \rightarrow A$ that can be expressed as a finite sum of two-sided multiplications, that is

$$
\phi(x)=\sum_{i} a_{i} x b_{i}
$$

for some finite collections of $a_{i}, b_{i} \in A$ (the coefficients of $\phi$ ). We denote the set of all elementary operators on $A$ by $\mathcal{E} \ell(A)$.

For a prime algebra $A$, by $M(A)$ and $Q_{s}(A)$ we respectively denote the multiplier algebra and the symmetric algebra of quotients of $A$ (see e.g. [1, 2]). The centre of $Q_{s}(A)$, denoted by $C(A)$, is called the extended centroid and it is a field [1, Corollary 2.1.9]. A unital prime algebra $A$ is said to be centrally closed if $C(A)=$ $Z(A)=\mathbb{F} 1$. In particular, a unital simple algebra is centrally closed if and only if it is central.

If $V$ is a vector space and $L$ a subspace of $V$, then a finite subset $\left\{v_{1}, \ldots, v_{n}\right\}$ of $V$ is said to be independent over $L$ if the set $\left\{v_{1}+L, \ldots, v_{n}+L\right\}$ is linearly independent in $V / L$.

We will frequently use the next two well-known facts, but as we have been unable to find a direct reference we include their proofs for completeness.

Lemma 2.1. Let $V$ and $W$ be vector spaces and let $L$ be a subspace of $V$. Assume that

$$
t=\sum_{i=1}^{n} v_{i} \otimes w_{i} \in V \otimes W
$$

is a tensor of rank $n \geq 1$ such that $v_{i} \notin L$ for some $1 \leq i \leq n$. Then there are $1 \leq k \leq n, v_{1}^{\prime}, \ldots, v_{n}^{\prime} \in V$ and $w_{1}^{\prime}, \ldots, w_{n}^{\prime} \in W$ such that

$$
t=\sum_{i=1}^{n} v_{i}^{\prime} \otimes w_{i}^{\prime}
$$

where $\left\{v_{1}^{\prime}, \ldots, v_{k}^{\prime}\right\}$ is independent over $L$ and $v_{k+1}^{\prime}, \ldots, v_{n}^{\prime} \in L$. 
Proof. Without loss of generality we may assume that $\left\{v_{1}, \ldots, v_{k}\right\}$ is a maximal subset of $\left\{v_{1}, \ldots, v_{n}\right\}$ that is independent over $L$. If $k=n$ we are done, so assume that $k<n$. Then for each $j=k+1, \ldots, n$ there is $f_{j} \in L$ and scalars $\lambda_{1 j}, \ldots, \lambda_{k j} \in$ $\mathbb{F}$ that are not all zero such that

$$
v_{j}=\sum_{i=1}^{k} \lambda_{i j} v_{i}+f_{j}
$$

Then

$$
t=\sum_{i=1}^{k} v_{i} \otimes\left(w_{i}+\sum_{j=k+1}^{n} \lambda_{i j} w_{j}\right)+\sum_{j=k+1}^{n} f_{j} \otimes w_{j}
$$

is a desired decomposition of $t$.

Proposition 2.2. Let $A$ and $B$ be algebras. If $I$ and $J$ are ideals of $A$ and $B$, respectively, with corresponding canonical maps $q_{I}: A \rightarrow A / I$ and $q_{J}: B \rightarrow B / J$, then the map

$$
q_{I} \otimes q_{J}: A \otimes B \rightarrow(A / I) \otimes(B / J), \quad \sum_{i} a_{i} \otimes b_{i} \mapsto \sum_{i}\left(a_{i}+I\right) \otimes\left(b_{i}+J\right)
$$

is an algebra epimorphism with $\operatorname{ker}\left(q_{I} \otimes q_{J}\right)=I \otimes B+A \otimes J$. In particular,

$$
(A \otimes B) /(I \otimes B+A \otimes J) \cong(A / I) \otimes(B / J),
$$

as algebras.

Proof. Obviously, $q_{I} \otimes q_{J}$ is an algebra epimorphism and $I \otimes B+A \otimes J \subseteq \operatorname{ker}\left(q_{I} \otimes q_{J}\right)$. For the reverse inclusion, assume that

$$
t=\sum_{i=1}^{n} a_{i} \otimes b_{i} \in A \otimes B
$$

is a tensor of rank $n \geq 1$ such that $\left(q_{I} \otimes q_{J}\right)(t)=0$. If all $a_{i}$ belong to $I$ and all $b_{i}$ belong to $J$ we have nothing to prove. Assume that some $a_{i} \notin I$. By Lemma 2.1 we may assume that $\left\{a_{1}, \ldots, a_{k}\right\}$ is independent over $I$ and $a_{k+1}, \ldots, a_{n} \in I$ for some $1 \leq k \leq n$. Then

$$
\sum_{i=1}^{k}\left(a_{i}+I\right) \otimes\left(b_{i}+J\right)=\left(q_{I} \otimes q_{J}\right)(t)=0,
$$

which forces $b_{1}, \ldots, b_{k} \in J$. Hence,

$$
t=\sum_{j=k+1}^{n} a_{j} \otimes b_{j}+\sum_{i=1}^{k} a_{i} \otimes b_{i} \in I \otimes B+A \otimes J
$$

The similar argument also shows that $t \in I \otimes B+A \otimes J$ if some $b_{i} \notin J$.

\section{RESUlts}

We begin this section with the next simple observation.

Proposition 3.1. Let $A$ be a nearly simple algebra. Then:

(a) $A$ is prime.

(b) $I_{A}$ is a simple infinite-dimensional algebra such that $Z\left(I_{A}\right)=\{0\}$.

(c) $Z(A)$ is a field and $Q_{s}(A)=M\left(I_{A}\right)$. 
Proof. (a) Since the only non-trivial ideal $I_{A}$ of $A$ satisfies $I_{A}^{2} \neq\{0\}, A$ is obviously prime.

(b) Assume that $J$ is a non-zero ideal of $I_{A}$. Since $A$ is prime, $I_{A}$ is also prime (see e.g. [1, Lemma 1.1.3]). In particular, $J^{3} \neq\{0\}$, so $I_{A} J I_{A}$ is a non-zero ideal of $A$ that is contained in $J$. Then, since $A$ is nearly simple, we have $I_{A}=I_{A} J I_{A} \subseteq J$, and thus $J=I_{A}$. This shows that $I_{A}$ is simple.

Assume that $I_{A}$ is unital with unity $e$. Then by [4, Lemma 2.54] $e$ is a central idempotent of $A$ such that $I_{A}=e A$. Then $(1-e) A$ is also a non-trivial ideal of $A$, so $I_{A}=(1-e) A$ and thus $I_{A}=\{0\}$; a contradiction. Hence, $I_{A}$ is a non-unital simple algebra and consequently $Z\left(I_{A}\right)=\{0\}$.

Now assume that $I_{A}$ is finite-dimensional. Then, since $I_{A}$ is simple, Wedderburn's Theorem (see e.g. [4, Theorem 2.61]) implies that there is a natural number $n$ and a division algebra $\mathbb{D}$ over $\mathbb{F}$ such that $I_{A} \cong \mathrm{M}_{n}(\mathbb{D})$. In particular, $I_{A}$ is unital; a contradiction with the preceding paragraph.

(c) Assume that $z \in Z(A)$ is a non-invertible element. Then $z A$ is an ideal of $A$ such that $z A \neq A$ and hence $z A \subseteq I_{A}$. In particular, by (b),

$$
z \in Z(A) \cap I_{A} \subseteq Z\left(I_{A}\right)=\{0\},
$$

that is, $z=0$. Thus, $Z(A)$ is a field. Finally, the equality $Q_{s}(A)=M\left(I_{A}\right)$ is a direct consequence of [1, Proposition 2.1.3] and the fact that $I_{A}^{2}=I_{A}$.

We now present some examples of nearly simple algebras.

Example 3.2. Let $A$ be a non-unital simple algebra. Then its unitization $A^{\sharp}=$ $\mathbb{F} \times A$ (see e.g. [4, Section 2.3]) is a nearly simple algebra with $I_{A^{\sharp}}=A$.

Similarly, set

$$
B:=C(A)+A \subseteq Q_{s}(A) .
$$

As $A$ is simple and non-unital, we have $Q_{s}(A)=M(A)$ and $C(A) \cap A=Z(A)=$ $\{0\}$. Hence, since $A$ is an ideal of $M(A), B$ is a subalgebra of $M(A)$ such that $B / A \cong C(A)$ (which is a field). Thus, $B$ is a nearly simple algebra with $I_{B}=A$.

Example 3.3. Let $V$ be a vector space over $\mathbb{F}$ of countably infinite dimension. Consider the algebra $\operatorname{End}_{\mathbb{F}}(V)$ of all linear operators on $V$. If by $\mathrm{F}(V)$ we denote the ideal of finite rank operators in $\operatorname{End}_{\mathbb{F}}(V)$, it is well-known that $\mathrm{F}(V)$ is the only non-trivial ideal of $\operatorname{End}_{\mathbb{F}}(V)$ and that $\operatorname{End}_{\mathbb{F}}(V)=M(\mathrm{~F}(V))$. In particular, by Proposition $3.1(\mathrm{c}), Q_{s}\left(\operatorname{End}_{\mathbb{F}}(V)\right)=\operatorname{End}_{\mathbb{F}}(V)$, so $\operatorname{End}_{\mathbb{F}}(V)$ is a nearly simple centrally closed algebra (see also [4, Example 7.28]).

Further, if $D$ is any simple subalgebra of $\operatorname{End}_{\mathbb{F}}(V)$ that contains the identity operator 1 (e.g. one of Weyl algebras), define

$$
A:=D+\mathrm{F}(V) \subseteq \operatorname{End}_{\mathbb{F}}(V) .
$$

As $D$ is simple, we have $D \cap \mathrm{F}(V)=\{0\}$, so $A / \mathrm{F}(V) \cong D$ is simple and thus $\mathrm{F}(V)$ is the unique non-trivial ideal of $A$. Hence, $A$ is a nearly simple algebra. Further, since by Proposition 3.1 (c) $Q_{s}(A)=M(\mathrm{~F}(V))=\operatorname{End}_{\mathbb{F}}(V), C(A)=Z\left(\operatorname{End}_{\mathbb{F}}(V)\right)=\mathbb{F} 1$, so $A$ is also centrally closed.

Definition 3.4. If $A$ and $B$ are nearly simple algebras, we say that an ideal $\mathcal{J}$ of $A \otimes B$ is admissible if $\mathcal{J}$ is either trivial or of the form as in (1.1).

Lemma 3.5. Assume that $A$ and $B$ are two nearly simple algebras such that all ideals of $A \otimes B$ are admissible. Then the algebra $\left(A / I_{A}\right) \otimes B$ is nearly simple, whose 
only non-trivial ideal is $\left(A / I_{A}\right) \otimes I_{B}$. Similarly, $A \otimes\left(B / I_{B}\right)$ is nearly simple with a non-trivial ideal $I_{A} \otimes\left(B / I_{B}\right)$.

Proof. Let $q_{I_{A}}: A \rightarrow A / I_{A}$ be the canonical map and consider the algebra epimorphism $q_{I_{A}} \otimes \operatorname{id}_{B}: A \otimes B \rightarrow\left(A / I_{A}\right) \otimes B$. Let $\mathcal{J}$ be a non-trivial ideal of $\left(A / I_{A}\right) \otimes B$. Then, since all ideals of $A \otimes B$ are admissible, and $\operatorname{ker}\left(q_{I_{A}} \otimes \operatorname{id}_{B}\right)=I_{A} \otimes B$ (Proposition 2.2), $\left(q_{I_{A}} \otimes \mathrm{id}_{B}\right)^{-1}(\mathcal{J})$ is an ideal of $A \otimes B$ that strictly contains $I_{A} \otimes B$ and so $I_{A} \otimes B+A \otimes I_{B} \subseteq\left(q_{I_{A}} \otimes \operatorname{id}_{B}\right)^{-1}(\mathcal{J})$. Also, since $\mathcal{J}$ is non-trivial, $\mathcal{J} \neq\left(A / I_{A}\right) \otimes B$, so $\left(q_{I_{A}} \otimes \operatorname{id}_{B}\right)^{-1}(\mathcal{J}) \neq A \otimes B$. Consequently,

$$
\left(q_{I_{A}} \otimes \operatorname{id}_{B}\right)^{-1}(\mathcal{J})=I_{A} \otimes B+A \otimes I_{B}
$$

and thus

$$
\begin{aligned}
\left(A / I_{A}\right) \otimes I_{B} & =\left(q_{I_{A}} \otimes \operatorname{id}_{B}\right)\left(I_{A} \otimes B+A \otimes I_{B}\right) \\
& =\left(q_{I_{A}} \otimes \operatorname{id}_{B}\right)\left(\left(q_{I_{A}} \otimes \operatorname{id}_{B}\right)^{-1}(\mathcal{J})\right) \\
& =\mathcal{J} .
\end{aligned}
$$

The similar argument also shows that $I_{A} \otimes\left(B / I_{B}\right)$ is the only non-trivial ideal of $A \otimes\left(B / I_{B}\right)$.

We now record some non-examples which helped us to conjecture the main result of this paper, Theorem 3.9 .

Example 3.6. Let $V$ be a real vector space of countably infinite dimension. Consider $\mathbb{C}$ as a unital subalgebra of $\operatorname{End}_{\mathbb{R}}(V)$. For example, if $\left\{e_{n}: n \in \mathbb{N}\right\}$ is a basis for $V$, define a linear operator $T \in \operatorname{End}_{\mathbb{R}}(V)$ by $T\left(e_{2 n-1}\right)=e_{2 n}$ and $T\left(e_{2 n}\right)=-e_{2 n-1}$ for all $n \in \mathbb{N}$. Then obviously $T^{2}=-1$, where 1 is the identity operator, so we can identify $\mathbb{C}$ with the subalgebra $\{\alpha 1+\beta T: \alpha, \beta \in \mathbb{R}\}$ of $\operatorname{End}_{\mathbb{R}}(V)$. Set

$$
A:=\mathbb{C}+\mathrm{F}(V) \subseteq \operatorname{End}_{\mathbb{R}}(V) .
$$

By Example $3.3 A$ is a centrally closed nearly simple (real) algebra with $I_{A}=\mathrm{F}(V)$. Consider the tensor product $A \otimes A$. Since by Proposition 2.2

$$
(A \otimes A) /(\mathrm{F}(V) \otimes A+A \otimes \mathrm{F}(V)) \cong(A / \mathrm{F}(V)) \otimes(A / \mathrm{F}(V)) \cong \mathbb{C} \otimes_{\mathbb{R}} \mathbb{C}
$$

and since $\mathbb{C} \otimes_{\mathbb{R}} \mathbb{C}$ is not a field, we conclude that $\mathrm{F}(V) \otimes A+A \otimes \mathrm{F}(V)$ is not a maximal ideal of $A \otimes A$. In fact, since $\mathbb{C} \otimes_{\mathbb{R}} \mathbb{C} \cong \mathbb{C} \oplus \mathbb{C}$ (see e.g. [4, Example 4.45]), $\mathrm{F}(V) \otimes A+A \otimes \mathrm{F}(V)$ is contained in two distinct maximal ideals of $A \otimes A$.

Example 3.7. Let $W$ be a complex vector space of countably infinite dimension. Consider the real algebra

$$
B:=\mathbb{R} 1+\mathrm{F}(W) \subseteq \operatorname{End}_{\mathbb{C}}(W),
$$

where 1 is the identity operator. Then $B$ is a central nearly simple algebra whose only non-trivial ideal is $\mathrm{F}(W)$. Note that $B$ is not centrally closed, since by Proposition 3.1 (c) $Q_{s}(B)=M(\mathrm{~F}(W))=\operatorname{End}_{\mathbb{C}}(W)$ and thus $C(B)=Z\left(\operatorname{End}_{\mathbb{C}}(W)\right)=\mathbb{C} 1$ (see also [4, Example 7.37]). Let $c_{1}$ and $c_{2}$ be elements of $\mathbb{C} \otimes_{\mathbb{R}} \mathbb{C}$ defined by $c_{1}:=1 \otimes i+i \otimes 1$ and $c_{2}:=1 \otimes i-i \otimes 1$. Then

$$
\mathcal{J}_{1}:=c_{1}(\mathrm{~F}(W) \otimes \mathrm{F}(W)) \quad \text { and } \quad \mathcal{J}_{2}:=c_{2}(\mathrm{~F}(W) \otimes \mathrm{F}(W))
$$

are two non-zero ideals of $B \otimes B$ such that $\mathcal{J}_{1} \mathcal{J}_{2}=\{0\}$. In particular, $\mathcal{J}_{1}$ and $\mathcal{J}_{2}$ are non-admissible and $B \otimes B$ is not even prime. 
Example 3.8. Consider the tensor product $A \otimes B$ of real algebras $A$ and $B$ from Examples 3.6 and 3.7. If $c_{1}, c_{2} \in \mathbb{C} \otimes_{\mathbb{R}} \mathbb{C}$ are as in Example 3.7, then using the isomorphism $A / \mathrm{F}(V) \cong \mathbb{C}$, we see that

$$
\mathcal{K}_{1}:=c_{1}((A / \mathrm{F}(V)) \otimes \mathrm{F}(W)) \quad \text { and } \quad \mathcal{K}_{2}:=c_{2}((A / \mathrm{F}(V)) \otimes \mathrm{F}(W))
$$

are two non-zero ideals of $(A / \mathrm{F}(V)) \otimes B$ such that $\mathcal{K}_{1} \mathcal{K}_{2}=\{0\}$. In particular, $(A / \mathrm{F}(V)) \otimes B$ is not prime, so by Lemma $3.5 A \otimes B$ has a non-admissible ideal.

We now state the main result of the paper.

Theorem 3.9. Let $A$ and $B$ be two nearly simple algebras. Then all ideals of $A \otimes B$ are admissible if and only if all tensor products

$$
Z\left(A / I_{A}\right) \otimes Z\left(B / I_{B}\right), \quad C(A) \otimes Z\left(B / I_{B}\right), \quad Z\left(A / I_{A}\right) \otimes C(B), \quad C(A) \otimes C(B)
$$

are fields.

Remark 3.10. In Examples 3.6, 3.7 and 3.8 (respectively), when considering $A \otimes A$, $B \otimes B$ and $A \otimes B$ (respectively), all tensor products in (3.1) are fields, except $Z\left(A / I_{A}\right) \otimes Z\left(A / I_{A}\right), C(B) \otimes C(B)$ and $Z\left(A / I_{A}\right) \otimes C(B)$ (respectively). This in particular demonstrates that none of the assumptions of Theorem 3.9 cannot be omitted (if $A$ and $B$ are algebras from Examples 3.6 and 3.7, then by symmetry $B \otimes A$ has a non-admissible ideal, $C(B) \otimes Z\left(A / I_{A}\right)$ is not a field, while $Z\left(B / I_{B}\right) \otimes$ $Z\left(A / I_{A}\right), Z\left(B / I_{B}\right) \otimes C(A)$ and $C(B) \otimes C(A)$ are fields).

The proof of Theorem 3.9 heavily relies on the main result of [10] (see also 9]) and its consequence which we state below.

Theorem 3.11. 10, Theorem] If $A$ and $B$ are prime algebras, then each non-zero ideal of $A \otimes B$ contains a non-zero elementary tensor if and only if $C(A) \otimes C(B)$ is a field.

Corollary 3.12. If $A$ and $B$ are unital simple algebras, then $A \otimes B$ is a simple algebra if and only if $Z(A) \otimes Z(B)$ is a field.

Proof. If $A \otimes B$ is simple, then $Z(A) \otimes Z(B) \cong Z(A \otimes B)$ (see e.g. [4, Corollary 4.32]) is a field.

Conversely, since both $A$ and $B$ are unital and simple, we have $Q_{s}(A)=A$ and $Q_{s}(B)=B$, so in particular $C(A)=Z(A)$ and $C(B)=Z(B)$. Hence if $Z(A) \otimes Z(B)$ is a field, then $A \otimes B$ is simple by [10, Corollary 1$]$.

Lemma 3.13. Let $A$ and $B$ be algebras and let $\mathcal{J}$ be an ideal of $A \otimes B$. If $\phi \in \mathcal{E} \ell(A)$ and $\psi \in \mathcal{E} \ell(B)$ are elementary operators, then $(\phi \otimes \psi)(\mathcal{J}) \subseteq \mathcal{J}$.

In particular, if both $A$ and $B$ are unital and $a \otimes b \in \mathcal{J}$ for some $a \in A$ and $b \in B$, then $\langle a\rangle \otimes\langle b\rangle \subseteq \mathcal{J}$.

Proof. Assume that $\phi=\sum_{i} M_{a_{i}, a_{i}^{\prime}}$ and $\psi=\sum_{j} M_{b_{j}, b_{j}^{\prime}}$ for some finite collections of $a_{i}, a_{i}^{\prime} \in A$ and $b_{j}, b_{j}^{\prime} \in B$. Then

$$
\phi \otimes \psi=\sum_{j} \sum_{i} M_{a_{i} \otimes b_{j}, a_{i}^{\prime} \otimes b_{j}^{\prime}} \in \mathcal{E} \ell(A \otimes B)
$$

and thus $(\phi \otimes \psi)(\mathcal{J}) \subseteq \mathcal{J}$. 
Next, assume that both $A$ and $B$ are unital. If $a \otimes b \in \mathcal{J}$ for some $a \in A$ and $b \in B$, then for any $x \in\langle a\rangle$ and $y \in\langle b\rangle$ there are elementary operators $\phi \in \mathcal{E} \ell(A)$ and $\psi \in \mathcal{E} \ell(B)$ such that $\phi(a)=x$ and $\psi(b)=y$. Hence,

$$
x \otimes y=(\phi \otimes \psi)(a \otimes b) \in \mathcal{J}
$$

and consequently $\langle a\rangle \otimes\langle b\rangle \subseteq \mathcal{J}$.

Proposition 3.14. Let $A$ and $B$ be unital prime algebras.

(a) If both $A$ and $B$ contain the smallest non-zero ideals $I$ and $J$, respectively, then $I \otimes J$ is the smallest non-zero ideal of $A \otimes B$ if and only if $C(A) \otimes C(B)$ is a field.

(b) If $M$ and $N$ are maximal ideals of $A$ and $B$, respectively, then $M \otimes B+A \otimes N$ is a maximal ideal of $A \otimes B$ if and only if $Z(A / M) \otimes Z(B / N)$ is a field.

Proof. (a) Assume that $C(A) \otimes C(B)$ is a field and let $\mathcal{J}$ be a non-zero ideal of $A \otimes B$. By Theorem 3.11, $\mathcal{J}$ contains a non-zero elementary tensor $a \otimes b$. By Lemma 3.13 we have $\langle a\rangle \otimes\langle b\rangle \subseteq \mathcal{J}$. By assumption, $I \subseteq\langle a\rangle$ and $J \subseteq\langle b\rangle$, so $I \otimes J \subseteq\langle a\rangle \otimes\langle b\rangle \subseteq \mathcal{J}$.

If, on the other hand, $C(A) \otimes C(B)$ is not a field, choose a non-zero non-invertible element $c \in C(A) \otimes C(B)$. Since $I$ is the smallest non-zero ideal of $A$, we have $C(A) I \subseteq A$. Similarly, $C(B) J \subseteq B$. Then, by the proof of [10, Theorem], $c(I \otimes J)$ defines a non-zero ideal of $A \otimes B$ that does not contain a non-zero elementary tensor. In particular, $c(I \otimes J)$ cannot contain $I \otimes J$.

(b) Obviously $M \otimes B+A \otimes N$ is a maximal ideal of $A \otimes B$ if and only if $(A \otimes B) /(M \otimes B+A \otimes N)$ is a simple algebra. Since by Proposition 2.2

$$
(A \otimes B) /(M \otimes B+A \otimes N) \cong(A / M) \otimes(B / N),
$$

by Corollary $3.12(A \otimes B) /(M \otimes B+A \otimes N)$ is simple if and only if $Z(A / M) \otimes Z(B / N)$ is a field.

In the proof of Theorem 3.9 we will use the next version of Amitsur's Lemma (see [2, Theorem 4.2.7]) which states that if $T_{1}, \ldots, T_{n}$ are linear operators between vector spaces $V$ and $W$ such that the vectors $T_{1}(x), \ldots, T_{n}(x)$ are linearly dependent for every $x \in V$, then a non-trivial linear combination of $T_{1}, \ldots, T_{n}$ has a finite rank.

We will also use the next fact, which was proved in [6] (see also [3, 5]).

Lemma 3.15. 6. Lemma 3.5] Let $\delta$ be a non-zero derivation of a simple algebra $D$. If $\delta$ has a finite rank, then $D$ is finite-dimensional.

Proof of Theorem 3.9. First assume that all ideals of $A \otimes B$ are admissible. Proposition 3.14 then implies that $C(A) \otimes C(B)$ and $Z\left(A / I_{A}\right) \otimes Z\left(B / I_{B}\right)$ are fields. Further, by Lemma 3.5, the only non-trivial ideal of $\left(A / I_{A}\right) \otimes B$ is $\left(A / I_{A}\right) \otimes I_{B}$. In particular, since $\left(A / I_{A}\right) \otimes I_{B}$ contains non-zero elementary tensors, by Theorem $3.11 Z\left(A / I_{A}\right) \otimes C(B)$ is a field. A similar argument also shows that $C(A) \otimes Z\left(B / I_{B}\right)$ must be a field.

Now assume that all tensor products in (3.1) are fields and let $\mathcal{J}$ be a non-zero ideal of $A \otimes B$. By Proposition 3.14 (a) we have $I_{A} \otimes I_{B} \subseteq \mathcal{J}$. Assume that

$$
I_{A} \otimes I_{B} \subsetneq \mathcal{J} \text {. }
$$

If $q_{I_{A}}: A \rightarrow A / I_{A}$ and $q_{I_{B}}: B \rightarrow B / I_{B}$ are the canonical maps, then one of the ideals

$$
\left(q_{I_{A}} \otimes \operatorname{id}_{B}\right)(\mathcal{J}) \subseteq\left(A / I_{A}\right) \otimes B \quad \text { or } \quad\left(\operatorname{id}_{A} \otimes q_{I_{B}}\right)(\mathcal{J}) \subseteq A \otimes\left(B / I_{B}\right)
$$


must be non-zero, since otherwise

$$
\begin{aligned}
\mathcal{J} & \subseteq \operatorname{ker}\left(q_{I_{A}} \otimes \operatorname{id}_{B}\right) \cap \operatorname{ker}\left(\operatorname{id}_{A} \otimes q_{I_{B}}\right)=\left(I_{A} \otimes B\right) \cap\left(A \otimes I_{B}\right) \\
& =I_{A} \otimes I_{B} .
\end{aligned}
$$

Assume that $\left(q_{I_{A}} \otimes \operatorname{id}_{B}\right)(\mathcal{J})$ is a non-zero ideal of $\left(A / I_{A}\right) \otimes B$. By assumption, $Z\left(A / I_{A}\right) \otimes C(B)$ is a field, so by Theorem $3.11\left(q_{I_{A}} \otimes \mathrm{id}_{B}\right)(\mathcal{J})$ contains a non-zero elementary tensor.

Let $n \geq 1$ be the smallest number with the property that there exists a tensor $t \in$ $\mathcal{J}$ of rank $n$ such that $\left(q_{I_{A}} \otimes \operatorname{id}_{B}\right)(t)$ is a non-zero elementary tensor in $\left(A / I_{A}\right) \otimes B$. We claim that $n=1$, so that $a \otimes b \in \mathcal{J}$ for some $a \in A \backslash I_{A}$ and $b \in B \backslash\{0\}$. In this case, $\langle a\rangle=A$ and $I_{B} \subseteq\langle b\rangle$, so by Lemma $3.13\langle a\rangle \otimes\langle b\rangle \subseteq \mathcal{J}$. In particular,

$$
A \otimes I_{B} \subseteq \mathcal{J} .
$$

In order to obtain a contradiction, assume that $n>1$. Let $t \in \mathcal{J}$ be any tensor of rank $n$ for which there exist $a^{\prime} \in A \backslash I_{A}$ and $b^{\prime} \in B \backslash\{0\}$ such that

$$
\left(q_{I_{A}} \otimes \operatorname{id}_{B}\right)(t)=\left(a^{\prime}+I_{A}\right) \otimes b^{\prime} .
$$

If $t$ is represented as

$$
t=\sum_{i=1}^{n} a_{i} \otimes b_{i},
$$

then obviously not all $a_{i}$ belong to $I_{A}$. By Lemma 2.1 we may assume that the set $\left\{a_{1}, \ldots, a_{k}\right\}$ is independent over $I_{A}$ and that $a_{k+1}, \ldots, a_{n} \in I_{A}$ for some $1 \leq k \leq n$. Also, since $t$ is of rank $n$, the set $\left\{b_{1}, \ldots, b_{n}\right\}$ is linearly independent. We first show that $k=1$. Indeed, by (3.3) and (3.4) we have

$$
\sum_{i=1}^{k}\left(a_{i}+I_{A}\right) \otimes b_{i}=\left(a^{\prime}+I_{A}\right) \otimes b^{\prime}
$$

in $\left(A / I_{A}\right) \otimes B$. Clearly, $b^{\prime} \in \operatorname{span}\left\{b_{1}, \ldots, b_{k}\right\}$, since otherwise the set $\left\{b^{\prime}, b_{1}, \ldots, b_{k}\right\}$ would be linearly independent and consequently $a_{1}, \ldots, a_{k} \in I_{A}$; a contradiction. Hence, there are scalars $\lambda_{1}, \ldots, \lambda_{k} \in \mathbb{F}$ such that

$$
b^{\prime}=\sum_{i=1}^{k} \lambda_{i} b_{i}
$$

Then, by (3.5),

$$
\sum_{i=1}^{k}\left(a_{i}-\lambda_{i} a^{\prime}+I_{A}\right) \otimes b_{i}=0,
$$

which forces $a_{i}-\lambda_{i} a^{\prime} \in I_{A}$ for all $i=1, \ldots, k$. Since the set $\left\{a_{1}, \ldots, a_{k}\right\}$ is independent over $I_{A}$, this is only possible if $k=1$, as claimed. Hence $a_{1} \notin I_{A}$, while $a_{2}, \ldots, a_{n} \in I_{A}$.

Further, without loss of generality we may assume that $a_{1}=1_{A}$. Indeed, since $a_{1} \notin I_{A}$, we have $\left\langle a_{1}\right\rangle=A$, so there is an elementary operator $\phi \in \mathcal{E} \ell(A)$ such that $\phi\left(a_{1}\right)=1_{A}$. For $2 \leq i \leq n$ set $a_{i}^{\prime}:=\phi\left(a_{i}\right)$. Then, $a_{2}^{\prime}, \ldots, a_{n}^{\prime} \in I_{A}$ and by Lemma 3.13 we have

$$
t^{\prime}:=1_{A} \otimes b_{1}+\sum_{i=2}^{n} a_{i}^{\prime} \otimes b_{i}=\left(\phi \otimes \operatorname{id}_{B}\right)(t) \in \mathcal{J} .
$$


Obviously, $\left(q_{I_{A}} \otimes \operatorname{id}_{B}\right)\left(t^{\prime}\right)=\left(1_{A}+I_{A}\right) \otimes b_{1} \neq 0$ in $\left(A / I_{A}\right) \otimes B$, so by minimality of $n, t^{\prime}$ is also a tensor of rank $n$. So if $a_{1} \neq 1_{A}$, we may substitute $t$ by $t^{\prime}$. Hence, in the sequel we assume that

$$
t=1_{A} \otimes b_{1}+\sum_{i=2}^{n} a_{i} \otimes b_{i} \in \mathcal{J}
$$

where $a_{2}, \ldots, a_{n} \in I_{A}$.

Set $\mathbb{K}:=C(A)$. By Proposition $3.1(\mathrm{c})$, we have $C(A)=Z\left(M\left(I_{A}\right)\right)$, so $\mathbb{K} I_{A} \subseteq$ $I_{A}$. Hence, we may consider $I_{A}$ as an algebra over $\mathbb{K}$. Without loss of generality we may assume that $\left\{a_{2}, \ldots, a_{l}\right\}$ is a maximal $\mathbb{K}$-independent subset of $\left\{a_{2}, \ldots, a_{n}\right\}$. Then for each $j=l+1, \ldots, n$ there are $\alpha_{i j} \in \mathbb{K}$ such that

$$
a_{j}=\sum_{i=2}^{l} \alpha_{i j} a_{i}
$$

so by (3.6)

$$
t=1_{A} \otimes b_{1}+\sum_{i=2}^{l} a_{i} \otimes b_{i}+\sum_{j=l+1}^{n}\left(\sum_{i=2}^{l} \alpha_{i j} a_{i}\right) \otimes b_{j} .
$$

We claim there is an element $x_{0} \in I_{A}$ such that the set $\left\{\left[a_{2}, x_{0}\right], \ldots,\left[a_{l}, x_{0}\right]\right\}$ is $\mathbb{K}$-independent. Indeed, if this set would be $\mathbb{K}$-dependent for all $x_{0} \in I_{A}$, then by Amitsur's Lemma there are $\beta_{2}, \ldots, \beta_{l} \in \mathbb{K}$ which are not all zero such that the inner derivation $\delta: I_{A} \rightarrow I_{A}$ defined by

$$
\delta(x):=\left[\beta_{2} a_{2}+\ldots+\beta_{l} a_{l}, x\right]
$$

has a finite rank. Since by Proposition 3.1 (b) $I_{A}$ is simple and infinite-dimensional, Lemma 3.15 implies that $\delta$ is zero. As $Z\left(I_{A}\right)=\{0\}$ (again by Proposition 3.1(b)), we conclude that

$$
\beta_{2} a_{2}+\ldots+\beta_{l} a_{l}=0 .
$$

Since the set $\left\{a_{2}, \ldots, a_{l}\right\}$ is $\mathbb{K}$-independent, this yields $\beta_{2}=\ldots=\beta_{l}=0$; a contradiction.

If $x_{0} \in I_{A}$ is an element from the preceding paragraph, by (3.7) we have

$$
\mathcal{J} \ni\left[t, x_{0} \otimes 1_{B}\right]=\sum_{i=2}^{l}\left[a_{i}, x_{0}\right] \otimes b_{i}+\sum_{j=l+1}^{n}\left(\sum_{i=2}^{l} \alpha_{i j}\left[a_{i}, x_{0}\right]\right) \otimes b_{j} .
$$

Since $\left\{\left[a_{2}, x_{0}\right], \ldots,\left[a_{l}, x_{0}\right]\right\}$ is $\mathbb{K}$-independent, by [2, Theorem 2.3.3] for each $i=$ $2, \ldots, l$ there is an elementary operator $\phi_{i} \in \mathcal{E} \ell\left(I_{A}\right)$ such that

$$
\phi_{i}\left(\left[a_{i}, x_{0}\right]\right) \neq 0 \quad \text { and } \quad \phi_{i}\left(\left[a_{j}, x_{0}\right]\right)=0 \quad \forall j \in\{2, \ldots, l\} \backslash\{i\} .
$$

Further, since $0 \neq \phi_{i}\left(\left[a_{i}, x_{0}\right]\right) \in I_{A}$ and since $I_{A}$ is simple (Proposition $3.1(\mathrm{~b})$ ), $I_{A} \phi_{i}\left(\left[a_{i}, x_{0}\right]\right) I_{A}$ is a non-zero ideal of $I_{A}$ and thus $I_{A} \phi_{i}\left(\left[a_{i}, x_{0}\right]\right) I_{A}=I_{A}$. Hence, for each $i=2, \ldots, l$ there is another elementary operator $\psi_{i} \in \mathcal{E} \ell\left(I_{A}\right)$ such that

$$
\psi_{i}\left(\phi_{i}\left(\left[a_{i}, x_{0}\right]\right)\right)=a_{i} .
$$

Set $\theta_{i}:=\psi_{i} \circ \phi_{i}$. Then $\theta_{i}$ is an elementary operator on $I_{A}$ such that

$$
\theta_{i}\left(\left[a_{i}, x_{0}\right]\right)=a_{i} \quad \text { and } \quad \theta_{i}\left(\left[a_{j}, x_{0}\right]\right)=0 \quad \forall j \in\{2, \ldots, l\} \backslash\{i\} .
$$


By extending $\theta_{i}$ to an elementary operator on $A$ (with the same coefficients), (3.8) and Lemma 3.13 imply

$$
\mathcal{J} \ni\left(\theta_{i} \otimes \operatorname{id}_{B}\right)\left(\left[t, x_{0} \otimes 1_{B}\right]\right)=a_{i} \otimes b_{i}+\sum_{j=l+1}^{n}\left(\alpha_{i j} a_{i}\right) \otimes b_{j},
$$

for each $i=2, \ldots, l$, so by (3.7)

$$
\mathcal{J} \ni \sum_{i=2}^{l}\left(a_{i} \otimes b_{i}+\sum_{j=l+1}^{n}\left(\alpha_{i j} a_{i}\right) \otimes b_{j}\right)=t-1_{A} \otimes b_{1} .
$$

Since $t \in \mathcal{J}$, we conclude that $1_{A} \otimes b_{1} \in \mathcal{J}$, which contradicts the assumption that $n>1$. In particular, (3.2) holds.

The similar arguments would also show that if $\left(\operatorname{id}_{A} \otimes q_{I_{B}}\right)(\mathcal{J})$ is a non-zero ideal of $A \otimes\left(B / I_{B}\right)$, then $I_{A} \otimes B \subseteq \mathcal{J}$.

Finally, if $\mathcal{J}$ strictly contains $I_{A} \otimes B$ or $A \otimes I_{B}$ (respectively), then the above proof shows that $\mathcal{J}$ also contains $A \otimes I_{B}$ or $I_{A} \otimes B$ (respectively). In any of those two cases we clearly have $I_{A} \otimes B+A \otimes I_{B} \subseteq \mathcal{J}$. Since, by assumption, $Z\left(A / I_{A}\right) \otimes Z\left(B / I_{B}\right)$ is a field, Proposition 3.14 (b) implies that $I_{A} \otimes B+A \otimes I_{B}$ is a maximal ideal of $A \otimes B$, which finishes the proof.

Corollary 3.16. Let $A$ be a nearly simple algebra. The following conditions are equivalent:

(i) For any nearly simple algebra $B$, all ideals of $A \otimes B$ are admissible.

(ii) All ideals of $A \otimes A$ are admissible.

(iii) $A$ is centrally closed and $A / I_{A}$ is central.

Proof. (i) $\Longrightarrow$ (ii). This is trivial.

(ii) $\Longrightarrow$ (iii). If $A$ would not be centrally closed, $C(A)$ would be a proper field extension of $\mathbb{F}$. But then $C(A) \otimes C(A)$ is not a field, so by Proposition 3.14 (a) $A \otimes A$ has a non-admissible ideal. Similarly, if $A / I_{A}$ is not central, then $Z\left(A / I_{A}\right) \otimes$ $Z\left(A / I_{A}\right)$ is not a field, so by Proposition 3.14 (b) $A \otimes A$ has a non-admissible ideal.

(iii) $\Longrightarrow$ (i). By assumption $C(A) \cong Z\left(A / I_{A}\right) \cong \mathbb{F}$, so the assertion follows directly from Theorem 3.9 .

Example 3.17. In view of Example 3.3, if $V$ is a vector space over $\mathbb{F}$ of countably infinite dimension, Corollary 3.16 in particular applies to algebras $\operatorname{End}_{\mathbb{F}}(V)$ and $D+$ $\mathrm{F}(V)$, where $D$ is any central simple subalgebra of $\operatorname{End}_{\mathbb{F}}(V)\left(\right.$ that $\operatorname{End}_{\mathbb{F}}(V) / \mathrm{F}(V)$ is central follows from [6, Proposition 2.9]).

\section{REFERENCES}

[1] P. Ara, M. Mathieu, Local Multipliers of $C^{*}$-algebras, Springer, London, 2003.

[2] K. I. Beidar, W. S. Martindale 3rd, A. V. Mikhalev, Rings with Generalized Identities, Marcel Dekker, Inc., 1995.

[3] J. Bergen, Derivations in prime rings, Canad. Math. Bull. 26 (1983), 267-270.

[4] M. Brešar, Introduction to Noncommutative Algebra, Universitext, Springer, 2014.

[5] M. Brešar, D. Eremita, The lower socle and finite rank elementary operators, Comm. Algebra 31 (2003), 1485-1497.

[6] M. Brešar, I. Gogić, Centrally Stable Algebras, J. of Algebra, 537 (2019), 79-97.

[7] P. L. Clark, Field Theory, lecture notes, University of Georgia (available at http://math.uga.edu/ pete/FieldTheory.pdf). 
[8] H. Haghighi, M. Tousi, S. Yassemi, Tensor product of algebras over a field, In: Commutative Algebra. Noetherian and non-Noetherian Perspectives, pp. 181-202, Springer, New York (2011).

[9] E. Jespers, E. R. Puczyłowski, On ideals of tensor products, J. of Algebra 140 (1991), 124130.

[10] W.K. Nicholson, J.F. Watters, On tensor products and extended centroids, Proc. Amer. Math. Soc. 88 (1983), 215-217.

Department of Mathematics, Faculty of Science, University of Zagreb, Bijenička 30, 10000 Zagreb, Croatia

E-mail address: ilja@math.hr 